\title{
STUDIES ON THE BURNING OF MICRO- AND NANOALUMINUM PARTICLE CLOUDS IN AIR
}

\section{P. Escot Bocanegra, V. Sarou-Kanian, D. Davidenko, C. Chauveau, and I. Gökalp}

\begin{abstract}
An experimental study has been conducted to determine flame propagation velocities in micro- $(6 \mu \mathrm{m})$ and nanosized $(250 \mathrm{~nm})$ aluminum particle clouds for various concentrations in air. The experimental results show faster flame propagation in nanoparticle cloud with respect to the microparticle flame. Maximum flame temperature has been measured using a high-resolution spectrometer operating in the visible range. Analysis of combustion residual shows that nanoparticle combustion is realized via the gaseous phase. A laminar flame model has been proposed based on this observation. Model parameters have been derived by fitting the experimental results.
\end{abstract}

\section{INTRODUCTION}

The available literature shows that $\mathrm{Al}$ nanopowder can significantly improve the combustion properties of some energetic materials, especially for propulsion applications. Solid propellants containing Alex nanopowders exhibit burning rates much higher (in some cases, by a factor of 5 to 20 higher) than the propellant formulations containing regular Al powder [1]. Further, hydroxyl-terminated polybutadiene (HTPB)-based solid-fuel formulations containing Alex or other nanopowders demonstrated the mass burning rates in a hybrid rocket up to $50 \%$ higher than the baseline formulations with regular $\mathrm{Al}$ powder [2]. These superior performances of the energetic compositions containing Al nanoparticles compared to the conventional microparticles are related to higher specific area and reactivity of nanopowders.

Several experimental and theoretical studies have been performed on the combustion of microparticles in laminar flames [3-5] as well as nanoparticles in a hot flow [5] or a shock tube [6]. These studies provided data on the flame speed, combustion temperature, and particle burning time. Several combustion models have been proposed for the particle oxidation in solid phase [7] and for particle

This is an Open Access article distributed under the terms of the Creative Commons Attribution-Noncommercial License 3.0, which permits unrestricted use, distribution, and reproduction in any noncommercial medium, provided the original work is properly cited. 
flames $[4,5,8]$. In spite of this effort, at present, there is no reliable theory on the nanoparticle combustion mechanism as well as on the flame propagation in a nanoparticle cloud.

In the present work, the flame propagation in an aluminum particle cloud in air is studied both experimentally and numerically. The experiment is aimed at the determination of the flame propagation velocity and flame temperature. Experimental studies are performed for micro- and nanoparticles under similar conditions. The experimental results are then used to elaborate a laminar flame model, which is finally applied for a numerical study.

\section{EXPERIMENTAL SETUP AND METHODS}

The experimental part of the work is related to the combustion of aluminum particles injected into a tube and ignited by an electric spark after the formation of a cloud. The experiments are conducted for two purposes: (1) to measure the flame front propagation velocities for various $\mathrm{Al}$ particle concentrations and different $\mathrm{Al}$ particle sizes, and (2) to measure the temperature of the gas phase during cloud combustion.

Figure 1 shows the experimental setup used for studying the combustion of particle clouds in air under normal conditions in terms of pressure and temperature. The combustion chamber is essentially a quartz tube (inner diameter

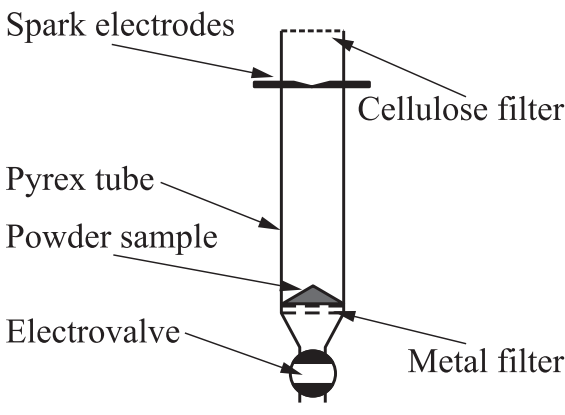

Figure 1 Combustion apparatus of $14 \mathrm{~mm}$, height of $180 \mathrm{~mm}$ giving an inner volume of $27.7 \mathrm{~cm}^{3}$ ), whose bottom end is closed by a porous metal plate, and the top end is closed by a thin cellulose filter. For the given reactor volume, the stoichiometric conditions in air correspond to $9.3 \mathrm{mg}$ of $\mathrm{Al}$ mass. The present experiments are performed for $\mathrm{Al}$ concentrations varying from 200 to $2000 \mathrm{~g} / \mathrm{m}^{3}$.

The powder sample is placed in the bottom of the tube. Suspension of particles and formation of a cloud inside the tube are achieved by a pulse air injection, using an electrical valve. The injection pressure and duration are adjusted to obtain a uniform distribution of particles inside the tube. After suspension, the cloud is stationary due to low sedimentation velocities, which are within the range of $0.01-1 \mathrm{~mm} / \mathrm{s}$ for $\mathrm{Al}$ nanoparticles. The particle cloud is ignited by a spark placed on the axis at $30 \mathrm{~mm}$ from the top of the tube. This ignition section was selected as 
providing the optimum ignition probability for all the combinations of the injected powder quantity, injection time, and pressure. A high-speed video camera (Phantom V5.0 with 3100 fps at a maximum resolution of $256 \times 1024$ pixels) is used for the observations of flame front propagation. The flame propagation velocity is determined by numerical image analysis.

For temperature measurements, flame emission spectra are recorded using a high-resolution spectrometer operating in the visible range with a resolution of $0.6 \mathrm{~nm}$. The measured spectra are divided by the apparatus function, obtained from a black body; then the continuous part, corresponding to the condensed phase emission, is subtracted to get the gas-phase spectra. Fitting of the measured and simulated intensity bands $\Delta \nu=-1,0,+1$ of the $\mathrm{AlO}$ emission is used for the determination of the maximum gas-phase temperature during cloud combustion. The simulation of the $\mathrm{AlO}$ emission spectra is based on the following theory [9]. Assuming that the medium is optically thick, the specific intensity is given by

$$
I_{\lambda}(T)=B_{\lambda}(T)\left[1-e^{-\alpha_{\lambda}^{\prime} l}\right] .
$$

The absorption coefficient corrected for stimulated emission is

$$
\alpha_{\lambda}^{\prime}=\frac{E_{\lambda}(T)}{B_{\lambda}(T)} .
$$

Assuming an optically thin medium, spontaneous emission is

$$
E_{\lambda}(T)=\frac{16 \pi^{3} c N_{u}(T) \nu_{\nu^{\prime} \nu^{\prime \prime}}^{4}}{3\left(2 J^{\prime}+1\right)} S_{\nu^{\prime} \nu^{\prime \prime}} S_{J^{\prime \prime} \Lambda^{\prime \prime}}^{J^{\prime} \Lambda^{\prime}} \cdot 10^{-11} .
$$

The number of $\mathrm{AlO}$ molecules in the upper state is

$$
N_{u}(T)=\frac{N\left(2 J^{\prime}+1\right)}{Q} e^{-E_{u} /(k T)}
$$

where $B_{\lambda}(T)$ is the Planck black body function; $l$ is the geometric path length; $c$ is the speed of light; $\nu_{\nu^{\prime} \nu^{\prime \prime}}$ is the frequency of emitted radiation; $\left(2 J^{\prime}+1\right)$ is the spin multiplicity; $S_{\nu^{\prime} \nu^{\prime \prime}}$ is the band strengths; $S_{J^{\prime \prime} \Lambda^{\prime \prime}}^{J^{\prime} \Lambda^{\prime}}$ is the line intensity factor; $N$ is the number density of molecules; $Q$ is the partition function; $E_{u}$ is the spontaneous emission in the upper state; $k$ is the Boltzmann constant; and $T$ is the temperature.

\section{EXPERIMENTAL RESULTS}

\subsection{Particle Visualization}

In the experiments, two different $\mathrm{Al}$ powders provided by the SNPE Matériaux Energétiques (SME) are used: a nanopowder $(250 \mathrm{~nm})$ and a micropowder 


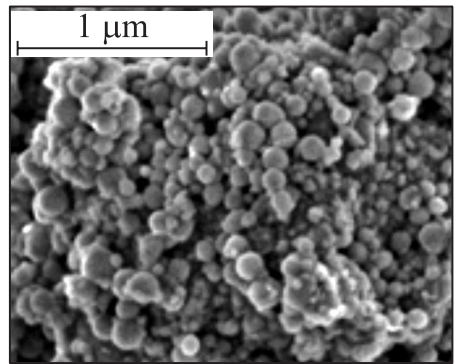

(a)

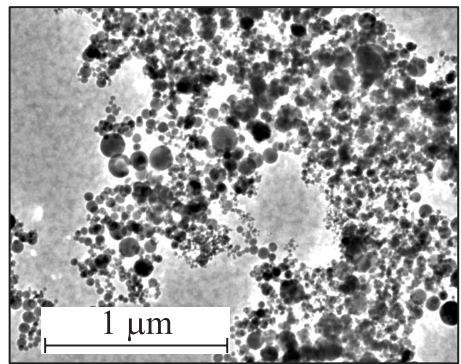

(b)

Figure 2 Scanning electron microscope visualization of initial nanopowder $(a)$ and TEM visualization of combustion residuals $(b)$

$(6 \mu \mathrm{m})$. The initial Al nanopowder visualized by a scanning electron microscope (SEM) is shown in Fig. $2 a$.

Residual particles of the same powder, collected after the combustion process, are visualized by a transmission electron microscope (TEM) in Fig. 2b. Mean Sauter diameters obtained from the images are 187 and $94 \mathrm{~nm}$, respectively. As the residual particle size is considerably smaller than the initial size, the combustion residuals are mainly formed not from solid-phase oxidation but from condensation of gas-phase reaction products. Residual particles obtained from the $\mathrm{Al}$ micropowder are almost of the same size.

\subsection{Flame Propagation Velocity}

The direct images show that after combustion initiation by a spark, the flame front propagates both downward and upward along the tube. The upward propagation ends quickly because it is accelerated by the hot product expansion. Then, only the downward propagating front remains whose position is used to determine the flame velocity. An example of the temporal evolution of the flame front position along the tube axis obtained by the numerical image analysis is presented in Fig. 3. The vertical axis represents the axial distance from the electrodes to the instantaneous flame front position. The curve shows two distinct stages in flame propagation. During the first stage, the flame front propagation is irregular. At the end of this stage, only the downward propagating flame remains in the tube. During the second stage, the flame propagation velocity stabilizes and becomes constant giving a constant curve slope $d x / d t$. A linear fit is used to determine the mean velocity on a time interval as shown in Fig. 3. It is supposed that the flame propagates at the fundamental velocity because the fresh mixture in front of it is at rest. Indeed, during combustion, the electric 


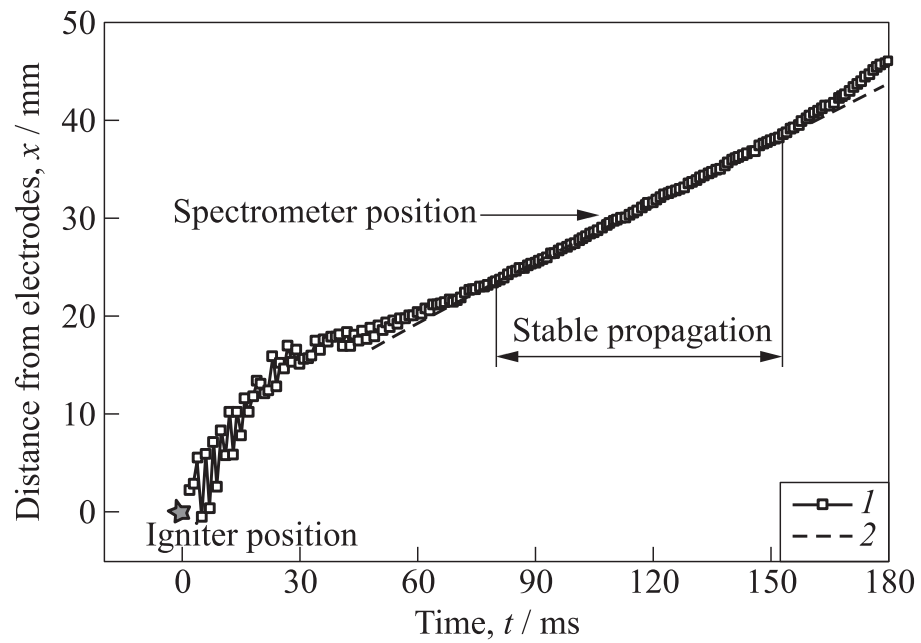

Figure 3 Temporal evolution of the flame front distance from the electrodes: 1 flame front position; 2 - velocity fit $(19.8 \mathrm{~cm} / \mathrm{s})$

valve is closed and the cellulose filter is opened by the hot gas ejection. It is found from the flame images that the flame front preserves its shape during the second stage. Together with the existence of the constant-velocity interval, this proves that the particle concentration is uniformly distributed along the tube.

Figure 4 presents the experimentally determined flame velocities vs. the particle concentration for micro- and nanoparticle clouds. The experimental points are obtained mainly for rich conditions: the equivalence ratio, $\phi$, is between 1.3 and 3.5 for microparticle flames and between 0.7 and 5 for nanoparticle flames. Outside these ranges, the flame initiation was not possible using the available spark system or flame propagation was unstable. Globally, the present results show that the flame propagation in nanoparticle clouds is faster at the same concentration. For the both particle sizes, the flame velocity has local maxima at $500-600 \mathrm{~g} / \mathrm{m}^{3}(\phi=1.6-1.9)$. Besides, nanoparticle flames exhibit a velocity increase at particle concentrations exceeding $850-950 \mathrm{~g} / \mathrm{m}^{3}(\phi>2.5-3)$. This behavior may have the following explanation. Normally, nanoparticles form agglomerates, which burn slower than individual particles; however, there is a certain quantity of relatively small agglomerates and individual particles. Under moderately rich conditions, the flame propagation depends mainly on the combustion properties of mid-size agglomerates and is limited by their burning rate. Under very rich conditions, the flame propagation is mainly governed by the combustion of small-size agglomerates and individual particles as their concentration becomes sufficient to consume the available oxygen. Experimental data on microparticle flames available from the literature $[3,4,10]$ are put in the 


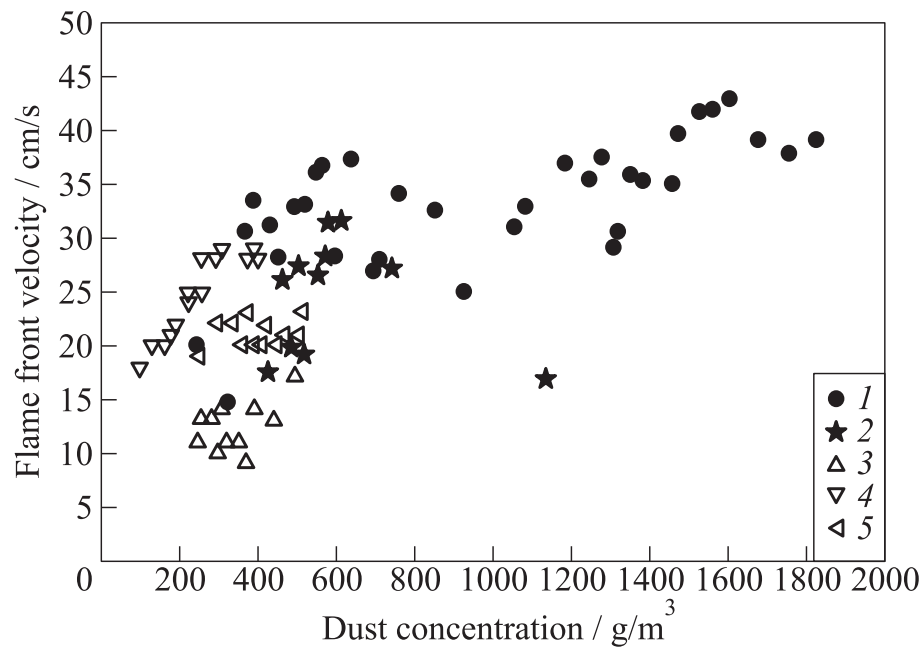

Figure 4 Flame velocity vs. dust (particle) concentration for different $\mathrm{Al}$ particle sizes: $1-250 \mathrm{~nm}$ (current study); $2-6 \mu \mathrm{m}$ (current study); $3-6 \mu \mathrm{m}$ [4]; $4-$ $6 \mu \mathrm{m}[10] ;$ and $5-5 \mu \mathrm{m}[3]$

same graph for comparison. The present data on the microparticle flames are in agreement with the experimental data by Risha et al. [4].

\subsection{Flame Temperature}

The AlO emission spectra were measured during the period of stable flame propagation as indicated in Fig. 3 by proper spectrometer positioning . The experimental and simulated $\mathrm{AlO}$ emission spectra are presented in Fig. 5 for micro- and nanoparticle flames. Well matched simulation spectra are obtained respectively for 3300 (Fig. $5 a$ ) and $2900 \mathrm{~K}$ (Fig. $5 b$ ) with a maximum uncertainty of $100 \mathrm{~K}$. These results are in good accordance with those from the literature $[4-6,11]$.

\section{LAMINAR FLAME MODEL FOR NANOPARTICLE CLOUD}

The present laminar flame model has been elaborated based on the experimental data presented in the previous section. The purpose is to obtain a simple model, which is controlled by few parameters that can be adjusted to match the experimental data on the flame propagation velocity and the maximum temperature in 


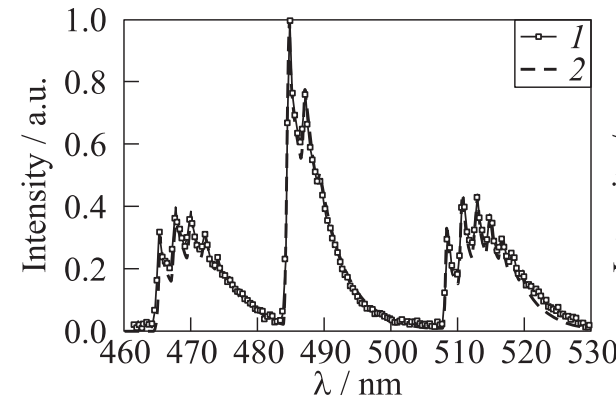

(a)

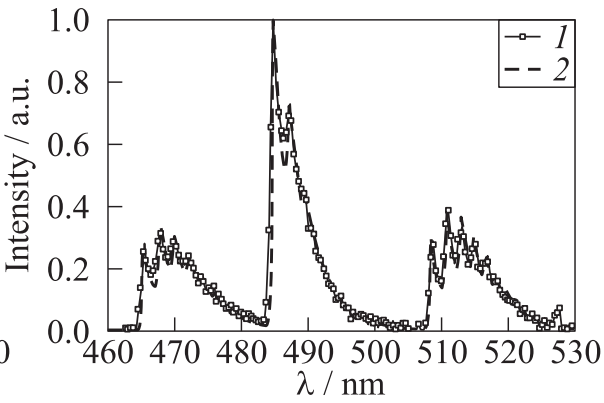

(b)

Figure 5 Experimental (1) and simulated (2) AlO emission spectra from microparticle $(a)$ and nanoparticle $(b)$ flames

the flame front. Note that this is not an attempt to simplify the understanding of the studied phenomenon. To the best of authors' knowledge, there is no reliable theory describing flame propagation in a nanoparticle cloud. One problem is related to a nanoparticle itself as its combustion properties cannot be uniquely characterized. They depend on the technology of particle production and passivation. Thus, experimental and theoretical results obtained for a particular kind of particles can be hardly accepted as applicable to any other particle kind. In addition, even if a reliable combustion model of an individual particle exists, it is not sufficient to build a flame model because of the following important reasons. Nanoparticles have strong tendency to agglomerate. It is not easy to determine the agglomerate structure in the cloud and to quantify the effect of the agglomeration on combustion and radiation properties with respect to the analogous properties of individual particles. Moreover, agglomeration depends on the kind of particles and on the way of creating particle cloud. In presence of agglomerates, the distribution of reactants in the cloud becomes significantly less uniform. Agglomerates can be macroscopically considered as large particles but their physicochemical properties can be very different.

The proposed laminar flame model consists of several submodels, which are considered in the following subsections.

\subsection{Particle Combustion Model}

From the comparative analysis of primary and residual particles (see Fig. 2), one can conclude that primary particles loose their mass during the combustion process. Thus, the shrinking core model [8] is not applicable. Residual or secondary particles can be formed by the oxide layer removal after its melting and by the 
condensation from the gas phase. This observation allows one to propose the following two-stage combustion model.

I Oxidation in the condensed phase when the temperature is greater than the melting temperature of $\mathrm{Al}, T_{\mathrm{Al}}^{f}$, and less than the melting temperature of $\mathrm{Al}_{2} \mathrm{O}_{3}, T_{\mathrm{Al}_{2} \mathrm{O}_{3}}^{f}$. Melting of $\mathrm{Al}$ core results in its expansion so the oxide layer may crack thus providing access of $\mathrm{O}_{2}$ to metallic Al. Besides, oxygen can diffuse through the oxide layer. The oxidation product, solid $\mathrm{Al}_{2} \mathrm{O}_{3}$, is supposed to deposit on the primary particle. Hence, during the first phase, particles gain mass and volume.

II Oxidation in the gas phase when the temperature exceeds $T_{\mathrm{Al}_{2} \mathrm{O}_{3}}^{f}$. It is supposed that after melting, the oxide layer forms one or more droplets that separate from the metallic core. These droplets partially represent secondary particles. This hypothesis is based on the fact that the residual particles have no apparent lobes and their size is significantly smaller than the initial particle size. The oxidation reaction proceeds in the gas phase and finally produces liquid $\mathrm{Al}_{2} \mathrm{O}_{3}$, for which two possible destinations can be proposed: deposition on existing secondary particles or forming new secondary particles of the same size. In the present model, the second option was preferred.

In principle, the third stage can be considered starting from the temperature of $\mathrm{Al}$ boiling. However, it is assumed to be globally included in the second stage.

The oxidation reaction is supposed to be always limited by processes occurring in the immediate vicinity of the metallic core. The diffusion process is not a limiting factor [6]. Hence, the overall oxidation rate must be proportional to the integral surface of particle cores. The rate of $\mathrm{Al}$ mass consumption is expressed by the following formulae:

$$
\dot{m}_{\mathrm{Al}}=\frac{d m_{\mathrm{Al}}}{d t}=-k \rho_{\mathrm{O}_{2} g} A_{\mathrm{Al}} N_{1}
$$

where $k$ is the reaction rate constant; $\rho_{\mathrm{O}_{2} g}$ is the partial density of $\mathrm{O}_{2}$ in the gas phase; $A_{\mathrm{Al}}$ is the surface area of the $\mathrm{Al}$ core; and $N_{1}$ is the number density of primary particles. The mass consumption rate is expressed in $\mathrm{kg} /\left(\mathrm{s} \cdot \mathrm{m}^{3}\right)$ and $k$ is expressed in $\mathrm{m} / \mathrm{s}$ and is supposed constant and independently specified for each oxidation stage. Thus, $k$ globally quantifies the reaction rate constant within the temperature range corresponding to a given oxidation stage. Moreover, it includes all real effects due to a particular size distribution, particle shape and passivation, particle agglomeration, etc.

The rates of $\mathrm{O}_{2}$ consumption and $\mathrm{Al}_{2} \mathrm{O}_{3}$ production are expressed as follows:

$$
\dot{m}_{\mathrm{O}_{2}}=f_{\mathrm{O}_{2}} \dot{m}_{\mathrm{Al}} ; \quad \dot{m}_{\mathrm{Al}_{2} \mathrm{O}_{3}}=-f_{\mathrm{Al}_{2} \mathrm{O}_{3}} \dot{m}_{\mathrm{Al}}
$$


where $f_{\mathrm{O}_{2}}$ and $f_{\mathrm{Al}_{2} \mathrm{O}_{3}}$ are the mass stoichiometric coefficients corresponding to the reaction

$$
\mathrm{Al}+\frac{3}{4} \mathrm{O}_{2} \Rightarrow \frac{1}{2} \mathrm{Al}_{2} \mathrm{O}_{3}
$$

\subsection{Thermodynamic and Solid-Liquid Transformation Models}

The reacting mixture is considered consisting of five species: (1) condensed aluminum, $\mathrm{Al}_{(S-L)}$; (2) solid aluminum oxide, $\mathrm{Al}_{2} \mathrm{O}_{3(S)}$; (3) liquid aluminum oxide, $\mathrm{Al}_{2} \mathrm{O}_{3(L)}$; (4) gaseous oxygen, $\mathrm{O}_{2}$; and (5) nitrogen, $\mathrm{N}_{2}$. Several modifications have been made to the standard thermodynamic data [12] in order to obtain a satisfactory model.

$\mathrm{Al}_{(S-L)}$ represents solid and liquid $\mathrm{Al}$. The temperature dependence of the enthalpy is modified to be continuous at $T=T_{\mathrm{Al}}^{f}$ including the latent heat of Al melting at $T>T_{\mathrm{Al}}^{f}$. Thus, the latent heat which is relatively small is not considered during the $\mathrm{Al}$ melting but is included in the overall reaction heat.

Standard thermodynamic properties of $\mathrm{Al}_{2} \mathrm{O}_{3(S)}$ and $\mathrm{Al}_{2} \mathrm{O}_{3(L)}$ are prolonged over $T_{\mathrm{Al}_{2} \mathrm{O}_{3}}^{f}$ because formally, the two species exist in the model irrespectively of the temperature. The entropy dependence is made discontinuous at $T=$ $T_{\mathrm{Al}_{2} \mathrm{O}_{3}}^{f}$ to obtain an abrupt change of the equilibrium constant, $K$, of the reaction $\mathrm{Al}_{2} \mathrm{O}_{3(S)} \Leftrightarrow \mathrm{Al}_{2} \mathrm{O}_{3(L)}$ by several orders of magnitude. This is a necessary measure providing a quick phase transformation process.

The standard temperature dependence of the specific heat of $\mathrm{Al}_{2} \mathrm{O}_{3(L)}$ is modified at $T \geq T_{\mathrm{Al}_{2} \mathrm{O}_{3}}^{f}$ to take into account the dissociation heat though the dissociation products are not explicitly included in the model. The modified dependence has been obtained from equilibrium computations using a more detailed chemical model [7].

The phase transformation process between $\mathrm{Al}_{2} \mathrm{O}_{3(S)}$ and $\mathrm{Al}_{2} \mathrm{O}_{3(L)}$ is formally described by the reaction $\mathrm{Al}_{2} \mathrm{O}_{3(S)} \Leftrightarrow \mathrm{Al}_{2} \mathrm{O}_{3(L)}$ whose backward rate constant is $k_{b}=10^{14} \mathrm{~mol} /\left(\mathrm{s} \cdot \mathrm{m}^{3}\right)$ and forward rate constant is determined as $k_{f}=K k_{b}$. This model provides the phase transformations in the flame front to be almost instantaneous with respect to the oxidation reaction. The rates of mass production/consumption due to the phase transformation are added to the respective oxidation rates.

\subsection{Radiation Model}

The particle cloud represents an optically thick medium whose emission and absorption properties must be taken into account. It is important to introduce a parameter controlling the radiation heat loss because the temperature profile and consequently the flame speed are sensitive to this factor. 
In the present model, the contributions of the radiation emission and absorption are not separated. Instead, these two contributions are described globally using an effective value of the particle emissivity.

The emissivities of primary and secondary particles are defined as follows:

$$
\varepsilon_{1}=\frac{\varepsilon_{\mathrm{Al}} V_{\mathrm{Al}}+\varepsilon_{\mathrm{Al}_{2} \mathrm{O}_{3}} V_{\mathrm{Al}_{2} \mathrm{O}_{3}}}{V_{\mathrm{Al}}+V_{\mathrm{Al}_{2} \mathrm{O}_{3}}} ; \quad \varepsilon_{2}=\varepsilon_{\mathrm{Al}_{2} \mathrm{O}_{3}}
$$

where $\varepsilon_{\mathrm{Al}}$ and $\varepsilon_{\mathrm{Al}_{2} \mathrm{O}_{3}}$ are the emissivities of $\mathrm{Al}$ and $\mathrm{Al}_{2} \mathrm{O}_{3}$ and $V_{\mathrm{Al}}$ and $V_{\mathrm{Al}_{2} \mathrm{O}_{3}}$ are the volumes of $\mathrm{Al}$ and $\mathrm{Al}_{2} \mathrm{O}_{3}$ in the primary particle.

The total radiation heat flux is expressed as:

$$
Q_{r}=-\sigma_{0}\left(\varepsilon_{1} A_{1} N_{1}+\varepsilon_{2} A_{2} N_{2}\right)\left(T^{4}-T_{\infty}^{4}\right)
$$

where $\sigma_{0}$ is the Stefan-Boltzmann constant; $A_{1}$ and $A_{2}$ are the surface areas of primary and secondary particles; $N_{1}$ and $N_{2}$ are the number densities of primary and secondary particles; and $T_{\infty}$ is the temperature at infinity.

To close the model, it is assumed that $\varepsilon_{\mathrm{Al}}=0.1$ whereas $\varepsilon_{\mathrm{Al}_{2} \mathrm{O}_{3}}$ is considered as an adjustable model parameter because its influence is more important.

\subsection{Transport Equations}

To formulate the transport equations describing the laminar flame, the following simplifying assumptions are adopted:

- the laminar flame is isobaric and freely propagating in an infinitely large space. The flame front is flat;

- scale separation: particle size and interparticle distance (microscale) are much smaller than the flame front thickness (macroscale). Thus, on the macroscale level, the two-phase mixture can be locally considered as homogeneous;

- infinitely fast microscale transport of mass, momentum, and energy: the gas and condensed phases move without slip and have the same temperature. On the microscale level, the composition of the gas phase is uniformly distributed;

- initially, all the particles are spherical and monodisperse. Their oxide layers are of the same initial thickness. During the oxidation, the growth of the oxide layer is uniform for all particles considered on the microscale level;

- particles are uniformly distributed. The number of primary particles transported per unit area per unit time is preserved across the flame front; 
- volume fraction of condensed phase is negligible with respect to the volume fraction of gas phase.

The laminar flame can be finally described by the following set of onedimensional (1D) transport equations in the moving frame of reference related to the flame front:

- energy conservation equation

$$
\rho \frac{\partial h}{\partial t}+G \frac{\partial h}{\partial x}=\frac{\partial}{\partial x}\left(\lambda \frac{\partial T}{\partial x}-\sum_{s} h_{s} J_{s}\right)+Q_{2}
$$

- species mass conservation equations

$$
\rho \frac{\partial Y_{s}}{\partial t}+G \frac{\partial Y_{s}}{\partial x}=-\frac{\partial J_{s}}{\partial x}+\dot{m}_{s}
$$

where $t$ is the time; $x$ is the spatial coordinate; $\rho$ is the mean density of two-phase mixture; $G$ is the mass flow rate per unit area; $h=\Sigma Y_{s} h_{s}$ is the mass specific enthalpy of mixture; $Y_{s}$ and $h_{s}$ are the mass fraction and mass specific enthalpy of species $s$, respectively; $\lambda$ is the thermal conductivity; and $J_{s}$ is the diffusion mass flux of species $s$.

The conservation of mixture mass is expressed as $G=\rho_{u} S_{L}$, where $\rho_{u}$ is the density of unburnt mixture and $S_{L}$ is the laminar flame speed. The momentum equation is replaced by the condition of constant pressure. The transport equations are written in the time-dependent formulation. The equation system is solved by the time integration until a steady-state solution is attained. The mass flow rate, $G$, is an eigenvalue of the problem providing a fixed flame front position in space. During time integration, the value of $G$ is adjusted to keep a given temperature on the temperature profile at a fixed coordinate.

The mean density of two-phase mixture is expressed by the following formula:

$$
\rho=\left(\frac{Y_{g}}{\rho_{g}}+\frac{Y_{c}}{\rho_{c}}\right)^{-1}
$$

with

$$
\begin{aligned}
Y_{g} & =Y_{\mathrm{O}_{2}}+Y_{\mathrm{N}_{2}} ; \\
Y_{c} & =Y_{\mathrm{Al}(S-L)}+Y_{\mathrm{Al}_{2} \mathrm{O}_{3}(S)}+Y_{\mathrm{Al}_{2} \mathrm{O}_{3}(L)} \\
\rho_{g} & =\frac{P}{R_{U} T} \frac{Y_{g}}{Y_{\mathrm{O}_{2}} / W_{\mathrm{O}_{2}}+Y_{\mathrm{N}_{2}} / W_{\mathrm{N}_{2}}} ; \\
\rho_{c} & =\frac{Y_{c}}{Y_{\mathrm{Al}(S-L)} / \rho_{\mathrm{Al}(S-L)}+Y_{\mathrm{Al}_{2} \mathrm{O}_{3}(S)} / \rho_{\mathrm{Al}_{2} \mathrm{O}_{3}(S)}+Y_{\mathrm{Al}_{2} \mathrm{O}_{3}(L)} / \rho_{\mathrm{Al}_{2} \mathrm{O}_{3}(L)}}
\end{aligned}
$$


where $P$ is the pressure; $R_{U}$ is the universal gas constant; and $W_{s}$ is the molar mass of species $s$.

The quantities $\lambda$ and $J_{s}$, related to the molecular transport, are evaluated only for the gas phase. Particles do not participate in the diffusion transport.

The present model has been realized as a computational code analogous to the PREMIX code of CHEMKIN-II [13]. It employs similar gas-phase models, grid-adaptation, and solution algorithm. However, the source code is totally different.

\section{NUMERICAL RESULTS}

The laminar flame model described above has three main parameters: reaction rate constants $k_{1}$ and $k_{2}$ for the two oxidation stages and effective emissivity $\varepsilon_{\mathrm{Al}_{2} \mathrm{O}_{3}}$. These parameters must be chosen to match the experimental data on flame propagation in a nanoparticle cloud. To find appropriate values for the three parameters, the following experimental data are taken as reference: mean Sauter diameter of primary particles of $187 \mathrm{~nm}$ and mean Sauter diameter of secondary particles of $94 \mathrm{~nm}$; particle mass concentration of $0.400 \pm 0.028 \mathrm{~kg} / \mathrm{m}^{3}$; flame propagation speed $S_{L}=0.32 \pm 0.04 \mathrm{~m} / \mathrm{s}$; maximum flame temperature $T_{\max }=3000 \pm 100 \mathrm{~K}$ that is $100 \mathrm{~K}$ larger than the temperature measured in the gas phase, because nanoparticles may have a higher temperature in the solid phase [6].

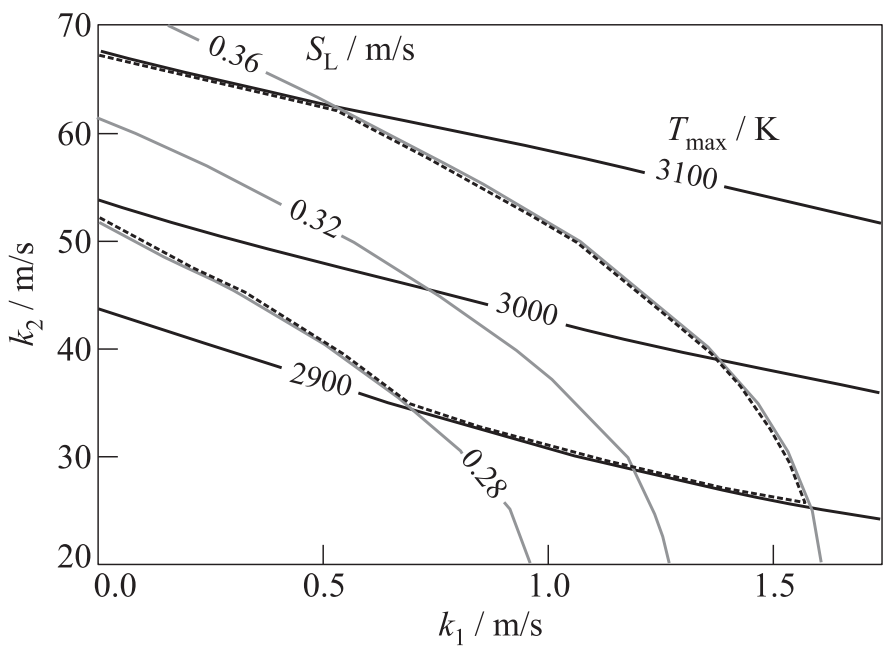

Figure 6 Contours of constant $S_{L}$ and $T_{\max }$ at $\varepsilon_{\mathrm{Al}_{2} \mathrm{O}_{3}}=0.3$ 


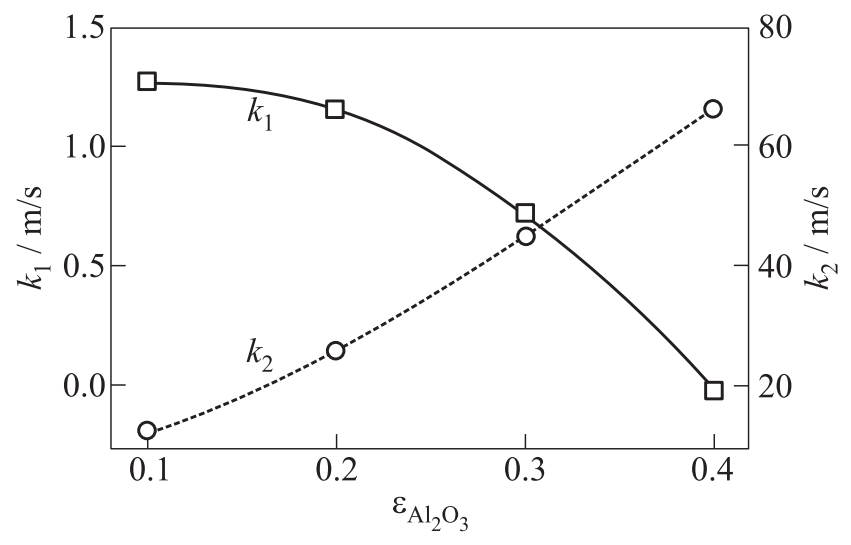

Figure 7 Reaction rate constants vs. emissivity

It is assumed that the initial thickness of oxide layer is $3 \mathrm{~nm}$ and the equivalence ratio, $\phi$, of fresh mixture is about 1.11. The following mass fractions are specified for the fresh mixture components: $Y_{\mathrm{Al}(S-L)}=0.2173, Y_{\mathrm{Al}_{2} \mathrm{O}_{3}}=0.0329$, $Y_{\mathrm{O}_{2}}=0.1743$, and $Y_{\mathrm{N}_{2}}=0.5756$.

Laminar flames have been simulated for the following parameter ranges: $k_{1}$ $=0-1.75 \mathrm{~m} / \mathrm{s}, k_{2}=5-80 \mathrm{~m} / \mathrm{s}$, and $\varepsilon_{\mathrm{Al}_{2} \mathrm{O}_{3}}=0.1-0.4$. Figure 6 shows the simulation results for $\varepsilon_{\mathrm{Al}_{2} \mathrm{O}_{3}}=0.3$. Contours of constant $S_{L}$ and $T_{\text {max }}$ are plotted in the plane $k_{1}-k_{2}$. Contours of each family represent the mean experimental value and the observed deviations from the mean value. Thus, the variation ranges of $k_{1}$ and $k_{2}$ can be identified by the limiting values of $S_{L}$ and $T_{\max }$ (dashed line in Fig. 6). The intersection of the contours $S_{L}=0.32 \mathrm{~m} / \mathrm{s}$ and $T_{\max }=3000 \mathrm{~K}$ corresponds to $k_{1}=0.729 \mathrm{~m} / \mathrm{s}$ and $k_{2}=45.5 \mathrm{~m} / \mathrm{s}$. Similar results have been obtained for different $\varepsilon_{\mathrm{Al}_{2} \mathrm{O}_{3}}$. The dependences of $k_{1}$ and $k_{2}$ on $\varepsilon_{\mathrm{Al}_{2} \mathrm{O}_{3}}$ are plotted in Fig. 7. Increase of $\varepsilon_{\mathrm{Al}_{2} \mathrm{O}_{3}}$ leads to greater heat losses, which are the most important at high temperatures. To keep the prescribed value of $T_{\max }$, the heat release during the second oxidation stage must be intensified by increasing $k_{2}$. The reaction heat is partially transferred upstream by the diffusion. This heat is added to the energy produced by the first oxidation stage, so to conserve the prescribed $S_{L}, k_{1}$ must be reduced. For the following analysis, $\varepsilon_{\mathrm{Al}_{2} \mathrm{O}_{3}}$ is taken equal to 0.3 and the corresponding values of $k_{1}$ and $k_{2}$ are fixed.

Another series of flames simulations has been made varying the initial particle diameter, $d_{p \text { ini }}$, and the equivalence ratio of the fresh mixture, $\phi$. The obtained results on $S_{L}$ and $T_{\max }$ are shown in Fig. 8. The graphs of $S_{L}$ indicate that the flame speed decreases with incleasing $d_{p \text { ini }}$, whereas its variation with $\phi$ is quite small for rich mixtures. These observations are in agreement with the experimentally observed tendencies. 


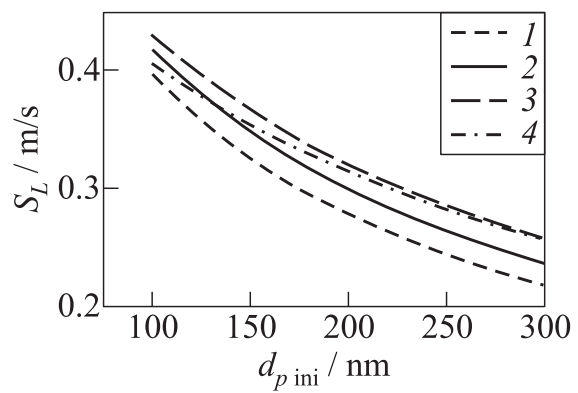

(a)

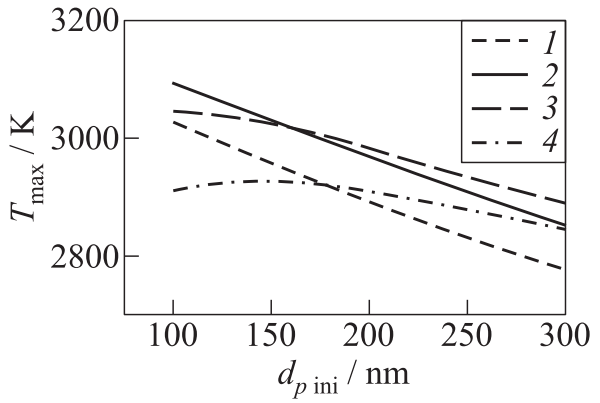

(b)

Figure 8 Predicted laminar flame speed $(a)$ and maximum temperature $(b)$ depending on the initial particle diameter for different equivalence ratios: $1-\phi=0.8 ; 2-$ $1 ; 3-1.5$; and $4-\phi=2$

Quantitatively, the influence of $d_{p \text { ini }}$ on $S_{L}$ seems to be more important than the experimental trend between the nano- and microparticle flames. The variation of $T_{\max }$ as a function of $d_{p \text { ini }}$ does not conform to the experimentally observed difference of flame temperature if one compares the nano- and microparticle flames. The variation of $T_{\max }$ with $\phi$ is within the experimental uncertainty.

Finally, the dependence of the particle burning time, $t_{b}$, on $d_{p \text { ini }}$ and $\phi$ is demonstrated in Fig. 9. The burning time is determined as the time period from the reaction start to the moment when $95 \%$ of combustible mass is consumed.

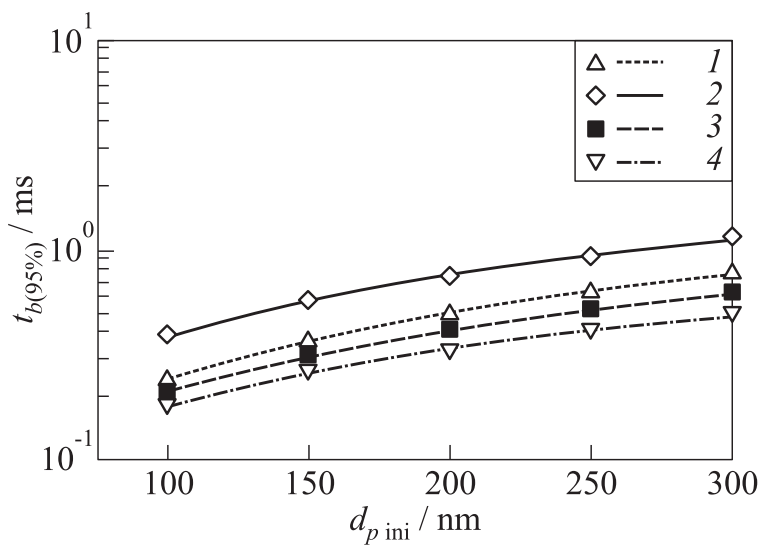

Figure 9 Predicted particle burning time vs. initial particle diameter for different equivalence ratios: $1-\phi=0.8 ; 2-1 ; 3-1.5$; and $4-\phi=2$ 
The computational results are presented along with the power fits $t_{b} \sim\left(d_{p \text { ini }}\right)^{n}$. The value of $n$ globally varies from 1.07 at $\phi=0.8$ to 0.92 at $\phi=2$. This dependence correlates with the existing theory [4] that the nanoparticle combustion is not limited by the diffusion process as in the case of microparticles, for which $n$ is close to 2 [5].

\section{CONCLUDING REMARKS}

The present results show that Al nanoparticle clouds burn faster than microparticle clouds for the same overall particle mass concentration in air. The flame propagation velocity depends also on the particle concentration and has different trends in the cases of micro- and nanoparticles: after a local maximum at $\phi=1.6-1.9$, the flame velocity in a nanoparticle cloud increases under very rich conditions $(\phi=3-5)$.

A laminar flame model for nanoparticle cloud in air has been proposed based on the experimentally observed facts. The model parameters have been fitted to match the experimental results on the flame propagation velocity and maximum temperature. This model conforms to the experimental behavior of the flame velocity and maximum temperature with respect to the equivalence ratio. It produces a nearly linear dependence of the burning time on the particle diameter. However, this model requires further improvement to correctly predict the effect of the particle diameter on the flame temperature and flame velocity.

\section{ACKNOWLEDGMENTS}

The authors acknowledge the support of SNPE-ME, Conseil Regional Center, and the CNRS.

\section{REFERENCES}

1. Ivanov, G. V., and F. Tepper. 1997. Activated aluminum as a stored energy source for propellants. A challenge in propellants and combustion, 100 years after Nobel. Ed. K. K. Kuo. New York: Begell House. 636-45.

2. Risha, G. A., A. Ulas, E. Boyer, S. Kumar, and K. K. Kuo. 2001. Combustion of HTPB-based fuels containing nano-sized energetic powder in a hybrid rocket motor. AIAA Paper No. 2001-3535.

3. Goroshin, S., I. Fomenko, and J.H. S. Lee. 1996. Burning velocities in fuel-rich aluminium dust clouds. Proc. Combust. Inst. 26:1961-67. 
4. Risha, G. A., Y. Huang, V. Yang, and R. A. Yetter. 2005. Analysis of nanoaluminum particle dust cloud combustion in different oxidizer. AIAA Paper No. 2005-0738.

5. Huang, Y., G. A. Risha, V. Yang, and R. A. Yetter. 2007. Combustion of bimodal nano/micro-sized aluminum particle dust in air. Proc. Combust. Inst. 31(2):2001-9.

6. Bazyn, T., H. Krier, and N. Glumac. 2007. Evidence for the transition from the diffusion-limit in aluminum particle combustion. Proc. Combust. Inst. 31(2):202128.

7. Trunov, M. A., M. Schoenitz, and E. L. Dreizin. 2006. Effect of polymorphic phase transformations in alumina layer on ignition of aluminum particles. Combust. Theory Modell. 10(4):603-23.

8. Aita, K., N. Glumac, S.P. Vanka, and H. Krier. 2006. Modelling the combustion of nano-sized aluminum particles. AIAA Paper No. 2006-1156.

9. Arnold, J. O., E.E. Whiting, and G.C. Lyle. 1969. Line by line calculation of spectra from diatomic molecules and atoms assuming a voigt line profile. J. Quant. Spectrosc. Radiat. Transfer 9(6):775-98.

10. Boichuk, L. V., V. G. Shevchuk, and A.I. Shvets. 2002. Flame propagation in twocomponent aluminum-boron gas suspensions. Combust. Explosion Shock Waves $38(6): 651-54$.

11. Goroshin, S., J. Mamen, A. Higgins, T. Bazyn, N. Glumac, and H. Krier. 2007. Emission spectroscopy of flame fronts in aluminum suspensions. Proc. Combust. Inst. 31(2):2011-19.

12. Burcat, A., and B. Ruscic. 2005. Third millennium ideal gas and condensed phase thermochemical database for combustion with updates from active thermochemical tables. Argonne National Laboratory, Technion. Report No. ANL-05/20, TAE 960.

13. Kee, R. J., J. F. Grcar, M. D. Smooke, and J. A. Miller. 1995. A Fortran program for modeling steady laminar one-dimensional premixed flames. Sandia National Laboratories. Sandia report SAND85-8240, UC-4. 